\title{
Laminar Validation Cases for the Incompressible Flow Model in ALE3D
}

\author{
J. Ortega
}

July 16, 2002

U.S. Department of Energy

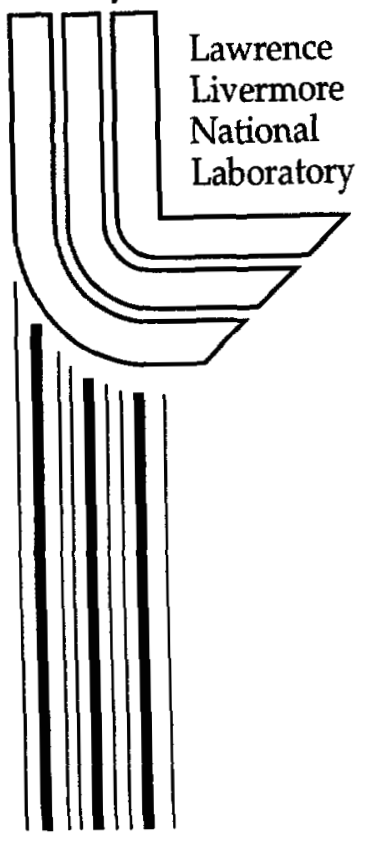




\section{DISCLAIMER}

This document was prepared as an account of work sponsored by an agency of the United States Government. Neither the United States Government nor the University of California nor any of their employees, makes any warranty, express or implied, or assumes any legal liability or responsibility for the accuracy, completeness, or usefulness of any information, apparatus, product, or process disclosed, or represents that its use would not infringe privately owned rights. Reference herein to any specific commercial product, process, or service by trade name, trademark, manufacturer, or otherwise, does not necessarily constitute or imply its endorsement, recommendation, or favoring by the United States Government or the University of California. The views and opinions of authors expressed herein do not necessarily state or reflect those of the United States Government or the University of California, and shall not be used for advertising or product endorsement purposes.

This work was performed under the auspices of the U.S. Department of Energy by the University of California, Lawrence Livermore National Laboratory under Contract No. W-7405-Eng-48.

This report has been reproduced directly from the best available copy.

Available electronically at http://www.doc.gov/bridge

Available for a processing fee to U.S. Department of Energy

And its contractors in paper from

U.S. Department of Energy

Office of Scientific and Technical Information

P.O. Box 62

Oak Ridge, TN 37831-0062

Telephone: (865) 576-8401

Facsimile: (865) 576-5728

E-mail: reports@adonis.osti.gov

Available for the sale to the public from

U.S. Department of Commerce

National Technical Information Service 5285 Port Royal Road

Springfield, VA 22161

Telephone: (800) 553-6847

Facsimile: (703) 605-6900

E-mail: orders@ntis.fedworld.gov

Online ordering: http://www.ntis.gov/ordering.htm

OR

Lawrence Livermore National Laboratory

Technical Information Department's Digital Library

http://www.llnl.gov/tid/Library.html 


\title{
Laminar Validation Cases for the Incompressible Flow Model in ALE3D
}

\author{
By
}

\author{
Jason Ortega \\ ortega17@llnl.gov
}

\subsection{Overview}

To benchmark the incompressible flow model in ALE3D ${ }^{1}$, two test cases are conducted. The first case of two-dimensional flow over a flat plate is selected because it provides a straightforward example to determine whether or not ALE3D can grow a boundary layer by viscous diffusion. The benefit of the flat plate problem is that under certain conditions, the governing Navier-Stokes equations can be simplified and solved with numerical techniques, providing an independent result that can be compared with the solution from ALE3D. The second test case is that of two-dimensional, laminar flow about a circular cylinder. This test case is selected because it provides the complexity of an unsteady bluff-body wake in which vorticity is periodically shed from the surface of the cylinder. Since this canonical flow problem has been studied extensively both experimentally and computationally, the results from ALE3D can be compared with those presented in the literature.

The results for the flat plate case demonstrate that the implicit time integration scheme results in an approximate twenty-four-fold reduction of the simulation time over that of the explicit time integration scheme. On the other hand, a problematic trend is observed in the explicit time integration scheme used in the flat plate case. The errors in both the velocity and shear stress are not reduced through grid refinement as one might expect. Another trend that raises concern with the flat plate problem is the sensitivity of the velocity and shear stress to the outlet zero natural boundary condition. In all of the flat plate simulations, at least one of the calculated quantities varies quite noticeably near the outlet of the flow domain. For the case of a circular cylinder in which an explicit time integration scheme is employed, both the drag coefficient and Strouhal number demonstrate trends of converging to a solution that compares favorably with results from other studies in the literature.

\subsection{Flow Over a Flat Plate}

Prandtl $^{2}$ was the first to demonstrate the simplifications that can be made to the Navier-Stokes equations for external flow over a body at high Reynolds number, Re. What follows is a brief summary of Prandtl's simplifications. Due to the no-slip boundary condition at the body, there exists a thin layer of fluid, called the boundary 
layer, in which the flow rapidly transitions from zero velocity at the wall to the free stream velocity, $U_{o}$, at the outer edge of the boundary layer. Consequently, steep velocity gradients $(\partial u / \partial y>>1)$ exist in this layer and, hence, even small amounts of viscosity can result in a relatively large wall shear stress, $\tau_{w}=\mu \partial u /\left.\partial y\right|_{y=0}$, where $\mu$ is the fluid viscosity and $y$ is the coordinate value in the normal direction away from the wall. Outside the boundary layer, the flow is irrotational and inertial forces dominate the flow field. With this physical reasoning, the steady-state, two-dimensional Navier-Stokes equations

$$
\begin{gathered}
u \frac{\partial u}{\partial x}+v \frac{\partial u}{\partial y}=-\frac{1}{\rho} \frac{\partial p}{\partial x}+v\left(\frac{\partial^{2} u}{\partial x^{2}}+\frac{\partial^{2} u}{\partial y^{2}}\right) \\
u \frac{\partial v}{\partial x}+v \frac{\partial v}{\partial y}=-\frac{1}{\rho} \frac{\partial p}{\partial y}+v\left(\frac{\partial^{2} v}{\partial x^{2}}+\frac{\partial^{2} v}{\partial y^{2}}\right) \\
\frac{\partial u}{\partial x}+\frac{\partial v}{\partial y}=0
\end{gathered}
$$

can be simplified by a scaling argument. It is assumed that $u \sim U_{o}, x \sim l, y \sim \delta, p \sim \rho U_{o}^{2}$, where $U_{o}$ is the free stream velocity, $l$ is the characteristic length of the body, $\delta$ is the boundary layer thickness $(\delta / l<1)$, and $\rho$ is the fluid density. Non-dimensionalizing the Navier-Stokes equations by these scaling factors and letting $R e_{x}=U_{0} x / v \gg 1$ reduces Eqs. 1-3 to

$$
\begin{gathered}
u \frac{\partial u}{\partial x}+v \frac{\partial u}{\partial y}=-\frac{1}{\rho} \frac{\partial p}{\partial x}+v \frac{\partial^{2} u}{\partial y^{2}} \\
\frac{\partial p}{\partial y}=0 \\
\frac{\partial u}{\partial x}+\frac{\partial v}{\partial y}=0
\end{gathered}
$$

for steady state flow. At the outer edge of the boundary layer, Eq. 4 becomes

$$
U_{o} \frac{\partial U_{o}}{\partial x}=-\frac{1}{\rho} \frac{\partial p}{\partial x}
$$

Thus, the pressure gradient can be obtained from the irrotational free stream velocity, $U_{o}$. From Eq. 5, this same pressure gradient exists through the entire boundary layer. Eq. 4 can be further simplified for flow over a flat plate. In this case, the free stream velocity is given by $U_{o}=$ const., such that $\partial p / \partial x=0$. Thus, as was shown by Blasius ${ }^{3}$, Eq. 4 reduces to 


$$
u \frac{\partial u}{\partial x}+v \frac{\partial u}{\partial y}=v \frac{\partial^{2} u}{\partial y^{2}}
$$

Introducing the similarity variable, $\eta=\frac{y}{\sqrt{x / U_{o}}}$ and the streamfunction, $\psi=\sqrt{U_{o} \nu x} f(\eta)$, Eqs. 6 and 7 reduce to a single third order, non-linear, ordinary differential equation,

$$
f f^{\prime \prime}+2 f^{\prime \prime \prime}=0
$$

where $f(\eta=0)=0, f^{\prime}(\eta=0)=0$, and $f^{\prime}(\eta \rightarrow \infty)=1$. Solving Eq. 9 numerically yields the horizontal and vertical velocity profiles shown in Figure 2.1. The skin friction coefficient, $C_{f}$, is given by

$$
C_{f}=\frac{\mu \partial u /\left.\partial y\right|_{y=0}}{\frac{1}{2} \rho U_{o}^{2}}=0.664 / \sqrt{\operatorname{Re}_{x}}
$$

It should be noted that neither the velocity profiles in Figure 2.1 nor $C_{f}$ is applicable near the leading edge of the plate since the assumption of $R e_{x} \gg>1$ is not valid for small $x$.

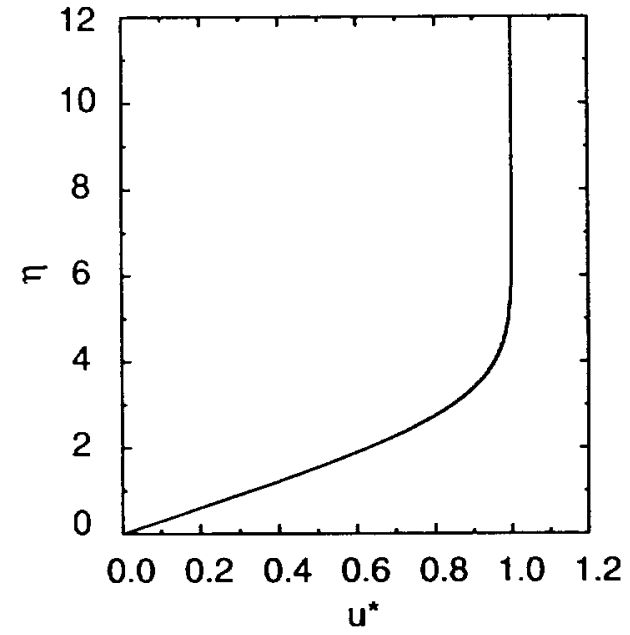

(a)

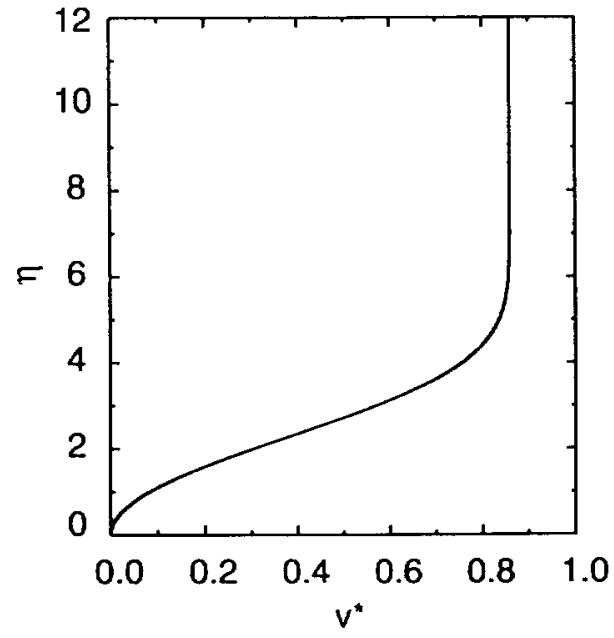

(b)

Figure 2.1. Horizontal and vertical velocity profiles from the numerical solution of Eq. 9 . In these plots, $u^{*}=f^{\prime}(\eta)$ and $v^{*}=v(\eta) / \sqrt{v U_{o} / x}=\frac{1}{2}\left(\eta f^{\prime}-f\right)$.

\subsection{Computational Setup}

Simulations of two-dimensional flow over a flat plate are performed with the incompressible flow model in ALE3D, which numerically solves the Navier-Stokes equations using a Galerkin finite element method. ${ }^{4.5}$ The ALE3D input files for these 
simulations are shown in Appendix 1. To determine the sensitivity of the results to the grid resolution, two grids, coarse and medium, are chosen for this exercise (Figure 2.2). Table 1 describes the details of these two grids. Both an explicit and an implicit timeintegration scheme are used in these simulations. The implicit scheme is used in this test case to determine its ability to decrease the simulation run time. Since the implicit scheme is not restricted by a numerically stable time step, it is capable of taking larger time steps than the explicit scheme. For the explicit simulations, the solution is advanced forward in time with a forward Euler time integration method. A projection method similar to that developed by Gresho $e t$ al. is used for the implicit solutions. ${ }^{4}$

A total of four simulations are performed for the flat plate test case: two simulations with the explicit scheme and two simulations with the implicit scheme. For the explicit scheme, the two simulations involve using the coarse and medium grids with the horizontal and vertical velocities specified at the inlet. The inlet velocity boundary conditions are chosen to be the horizontal and vertical velocity profiles of a Blasius boundary layer for $x_{o}=1.0, U_{o}=1.0$, and $v=0.0005$, effectively giving an inlet Reynolds number of 2,000 . Since $R e>>1$, the results of these simulations can be compared to the solution of the Blasius boundary layer problem. For the first simulation with the implicit scheme, only the horizontal velocity is specified at the inlet, while for the second simulation, both the horizontal and vertical velocities are specified at the inlet. This variation in the inlet boundary condition is done to determine the sensitivity of the solution to the inlet vertical velocity. When the vertical velocity component is left unspecified at the inlet, a zero natural boundary condition ${ }^{1}$ on the tangential traction is used at the inlet. Only the medium grid is used for the implicit simulations. Again, the inlet velocity profiles are taken to be those of a Blasius boundary layer for for $x_{o}=1.0, U_{o}$ $=1.0$, and $v=0.0005$ for the implicit simulations. The following boundary conditions are common to both the explicit and implicit time-integration simulations. At the bottom wall $(y=0)$, the velocity boundary condition is one of no-slip and no-penetration. A zero natural boundary condition is imposed at the top and outlet of the computational domain. The out-of-plane velocity component, $w$, is set to zero for all of the nodes. No boundary conditions are needed for the pressure. The initial conditions at $t=0$ are $u\left(x-x_{o}>, y>0\right)=$ 1.0 and $v\left(x-x_{o}>0, y>0\right)=0$. Internal to the code, this initial condition then is projected to a divergence-free field. ${ }^{4}$ Table 2 summarizes the details of each of the four simulations. The CFL number is taken to be 0.5 for both grids in the explicit simulations.

In the implicit scheme, the CFL number for the advection term is set to 3.0 , while the stability number for the diffusion term is set to $10^{6}$. Consequently, the time step in the implicit scheme simulations is not diffusion limited. For the explicit simulations, the governing equations are solved with a conjugate gradient solver with a tolerance set to $10^{-12}$. For the implicit simulations, a conjugate gradient solver is used to solve the pressure Poisson equation and a generalized minimum residual solver (gmres) is used to solve the momentum equations. For both of these solvers, the tolerance is set to $10^{-12}$. Since the vertical velocity field converges more slowly than the horizontal velocity field, 


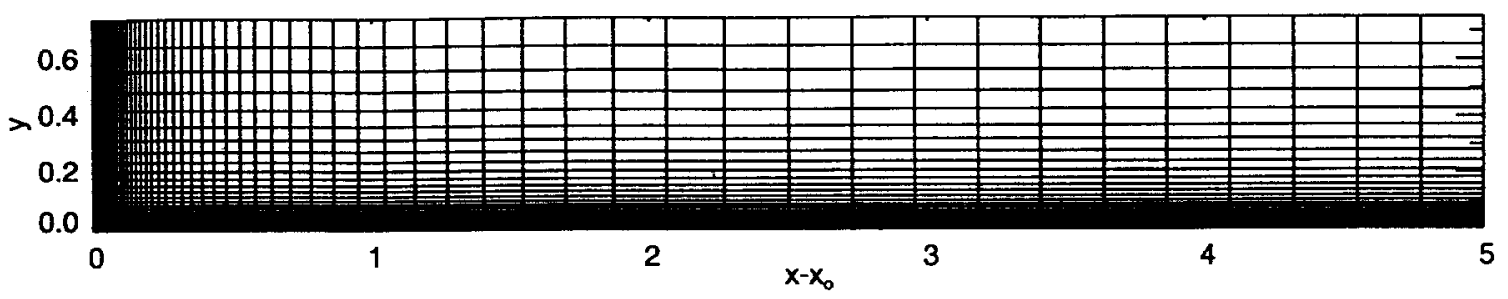

(a)

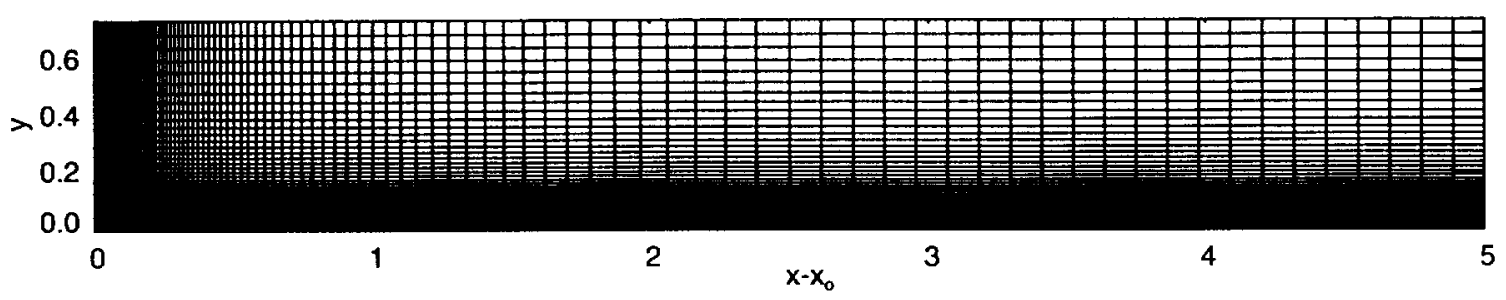

(b)

Figure 2.2. (a) Coarse and (b) medium grids used to simulate flow over a flat plate. The variable $x_{o}=1.0$ is the offset of the computational domain from origin of the $x$ axis.

\begin{tabular}{|c|c|c|c|c|c|}
\hline Grid & $\begin{array}{c}\text { Total } \\
\text { Number of } \\
\text { Elements }\end{array}$ & $\begin{array}{c}\text { Number } \\
\text { of } \\
\text { Elements } \\
\text { in } x\end{array}$ & $\begin{array}{c}\text { Number } \\
\text { of } \\
\text { Elements } \\
\text { in } y\end{array}$ & $\Delta x_{\min }$ & $\Delta y_{\min }$ \\
\hline Coarse & 2440 & 61 & 40 & 0.002152468 & 0.000419352 \\
\hline Medium & 9760 & 122 & 80 & 0.001050609 & 0.000202344 \\
\hline
\end{tabular}

Table 1. Details of the two grids used for the simulations of flow over a flat plate. The resolution is doubled in the $x$ and $y$ directions from the coarse to medium grid, such that the medium grid has four times as many elements as the coarse grid.

\begin{tabular}{|c|c|c|c|}
\hline Run & $\begin{array}{c}\text { Time-integration } \\
\text { scheme }\end{array}$ & $\mathrm{u}_{\text {inlet }}$ & $\mathrm{v}_{\text {inlet }}$ \\
\hline 1 & Explicit & Specified & Specified \\
\hline 2 & Explicit & Specified & Specified \\
\hline 3 & Implicit & Specified & Unspecified \\
\hline 4 & Implicit & Specified & Specified \\
\hline
\end{tabular}

Table 2. Details of the four flat plate simulations. 
the simulations are run until the absolute value of the relative change in the vertical velocity is less than $0.01 \%$. Each of the simulations in Runs 1-3 are done on eight 375 $\mathrm{MHz}$ processors on an IBM SP machine. On the other hand, Run 4 is done on only one processor, making a direct timing comparison with Run 2 somewhat difficult. However, if the timing performance of Run 2 is simply multiplied by 8 , it can be shown that the implicit time integration scheme runs approximately twenty-four times faster than the explicit time integration scheme for the case in which both the horizontal and vertical velocity components specified at the inlet. Post-processing and plotting of these simulations are done with Interactive Data Language (IDL) of Research Systems, Inc.

\subsection{Results and Discussion}

Plots of the horizontal velocity profiles and their relative differences with the Blasius horizontal velocity are shown in Figure 2.3. For both explicit cases (Runs 1-2), the maximum relative error in the horizontal velocity is approximately $1 \%$. As the grid is refined from Run 1 to Run 2, the reduction in the maximum error of the horizontal velocity is small. This trend indicates that the solution is fairly insensitive to the grid resolution and that further grid refinement may not reduce the error in the horizontal velocity significantly below $1 \%$. The error is somewhat smaller for the implicit simulations (Runs 3-4). For Run 3, the relative error is about $0.25 \%$, while it is approximately $0.5 \%$ for Run 4 . The profiles for the implicit cases exhibit noticeable dependence upon the chosen downstream location at which the profiles are taken, perhaps indicating sensitivity to the zero natural outlet boundary condition. Note that for Runs 1-2, and 4, in which both velocity components are specified at the inlet, ALE3D under predicts the horizontal velocity. However, for Run 3, in which only the horizontal velocity is specified at the inlet, ALE3D over predicts the values of the horizontal velocity. It can also be seen that specifying the vertical velocity in Run 4 results in a slightly greater error in the horizontal velocity than that in Run 3, where the inlet vertical velocity is unspecified.

The vertical velocity profiles (Figure 2.4 ) exhibit errors that are somewhat larger than those for the horizontal profiles. This observation is expected because the vertical velocity scales as $R e_{x}^{-1 / 2}$, making it significantly smaller than the horizontal velocity and more sensitive to errors in the numerical solution. For all four runs, the magnitude of the maximum error in the vertical velocity is approximately $3-4 \%$. In addition, the errors for both the explicit and implicit time integration schemes are dependent upon the downstream location at which the comparison with the Blasius profile is made. The magnitude of the error in the vertical velocity is consistently greatest at the farthest downstream location, perhaps indicating sensitivity to the outlet zero natural boundary condition. For the explicit simulations, the trend is for the Navier-Stokes computations to overshoot the correct values in the vertical velocity. On the other hand, the NavierStokes computations undershoot the correct vertical velocity for both implicit simulations. As is the case for the horizontal profiles, grid refinement in Runs 1 and 2 does not result in a reduction of the vertical velocity error. Again, this trend is problematic since it suggests that further grid refinement may not result in further error reduction. 
The shear stress coefficients and their relative errors are plotted in Figure 2.5. The computed values of $C_{f}$ follow the general trend of Eq. 10. Though, it can be seen that the relative error in the shear stress coefficient has a maximum absolute value of approximately $2-4 \%$ over all four simulations. For the explicit cases, the relative error decreases from zero at the inlet and then exhibits oscillations near the outlet. Once again, the error in the shear stress coefficient does not decrease as the grid is refined in Runs 1 and 2. For the implicit cases, the relative error in $C_{f}$ increases away from the inlet, decreases to a minimum, and then increases near the outlet. Unlike $C_{f e r r}$ for the explicit runs, $C_{\text {ferr }}$ for the implicit runs are relatively smooth and do not exhibit oscillations. Note, however, that there is a sudden drop in the relative error at the outlet for Run 3.

Analysis of the pressure indicates how well the Navier-Stokes computations represent the irrotational flow outside the boundary layer. Recall that for Blasius flow over a flat plate, $\partial p / \partial x=0$. Figure 2.6 shows plots of $p(x, y \approx 0.5)$ for the four simulations. Although the pressure varies over the computational domain, it can be seen that the horizontal gradient in the pressure is much less than $I$ for all four cases. It is interesting to note that for the cases in which both the horizontal and vertical velocity are specified at the inlet, the pressure at $x-x_{o}=0$ has a value of order $10^{-3}$. On the other hand, for the case in which only the horizontal velocity is specified at the inlet, the pressure is approximately $10^{-6}$ at $x-x_{o}=0$. 

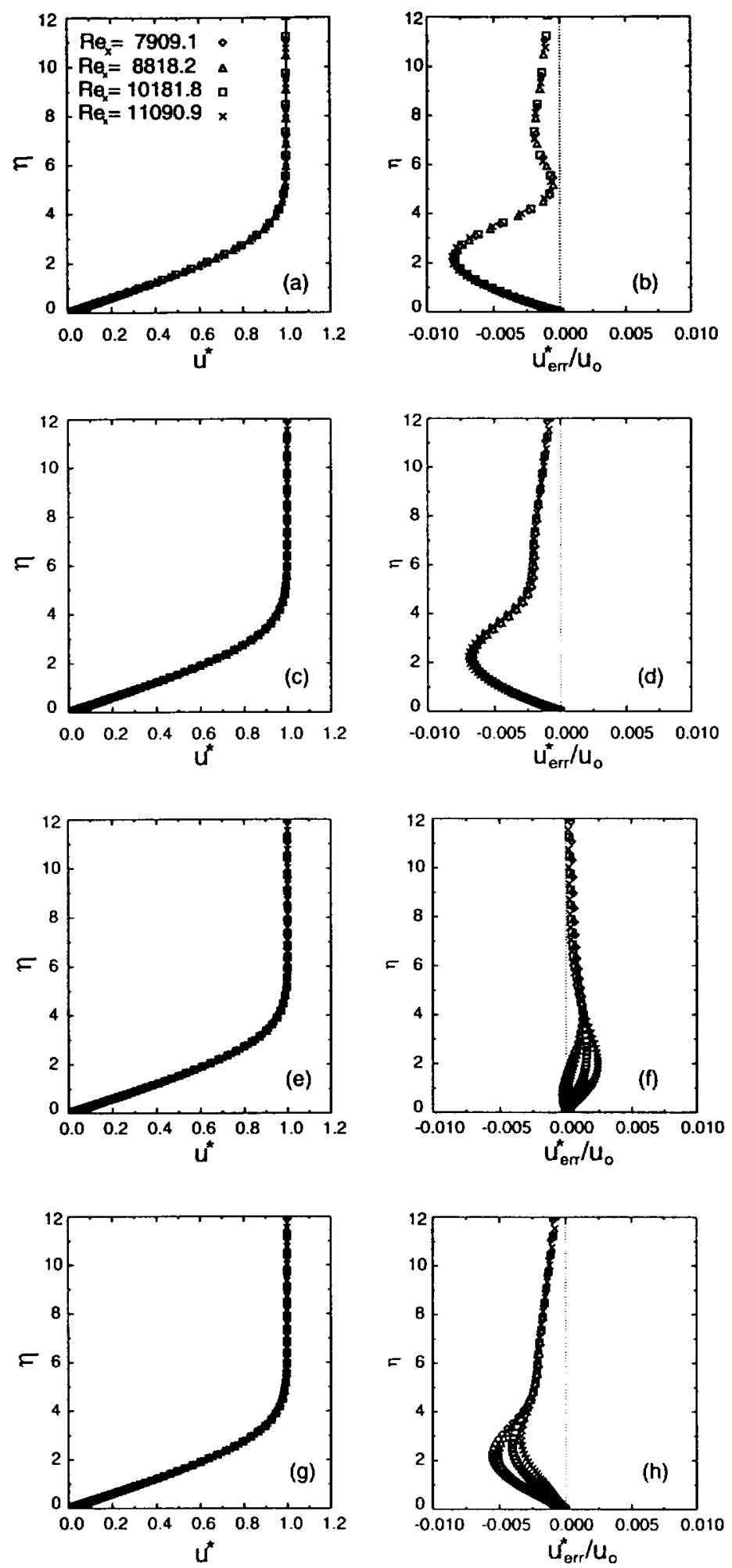

Figure 2.3. Horizontal velocity profiles at various $\operatorname{Re}_{x}$ for (a) Run 1, (c) Run 2, (e) Run 3, and (g) Run 4. Relative difference, $u_{e r r}^{*} / u_{o}=\left(u^{*}{ }_{A L E 3 D^{-} u^{*}}\right) / u_{o}$, between the computed horizontal velocity and the Blasius profile for (b) Run 1, (d) Run 2, (f) Run 3, and (h) Run 4 , where $u_{o}=1$. The solid line in $(\mathrm{a}, \mathrm{c}, \mathrm{e}, \mathrm{g})$ is $u^{*}$. 



Figure 2.4. Vertical velocity profiles at various $\operatorname{Re}_{x}$ for (a) Run 1, (c) Run 2, (e) Run 3, and (g) Run 4. Relative difference, $v_{e r r}^{*} / v_{o}=\left(v^{*}{ }_{A L E 3 D^{-}} v^{*}\right) / v_{o}$, between the computed horizontal velocity and the Blasius profile for (b) Run 1, (d) Run 2, (f) Run 3, and (h) Run 4 , where the variable $v_{o}=0.860853$ is the value of the vertical velocity outside the boundary layer. The solid line in $(\mathrm{a}, \mathrm{c}, \mathrm{e}, \mathrm{g})$ is $v^{*}$. 

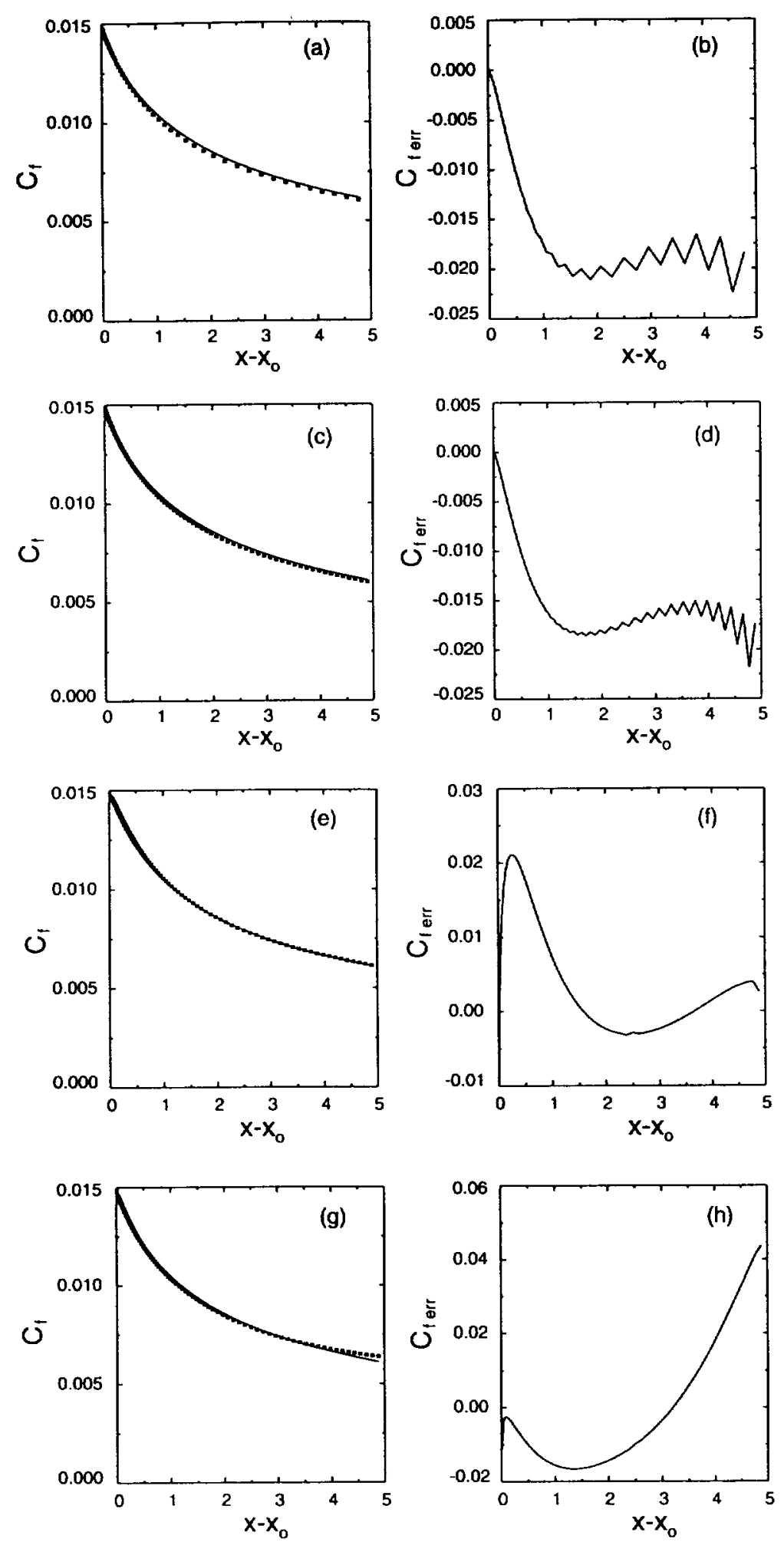

Figure 2.5. Shear stress coefficient for (a) Run 1, (c) Run 2, (e) Run 3, and (g) Run 4. Relative difference, $C_{f e r r} / C_{f}=\left(C_{f A L E 3 D}-C_{f}\right) / C_{f}$, between the computed shear stress coefficient and that of the Blasius solution for (b) Run 1, (d) Run 2, (f) Run 3, and (h) Run 4, The solid line in $(\mathrm{a}, \mathrm{c}, \mathrm{e}, \mathrm{g})$ is $C_{f}$. 

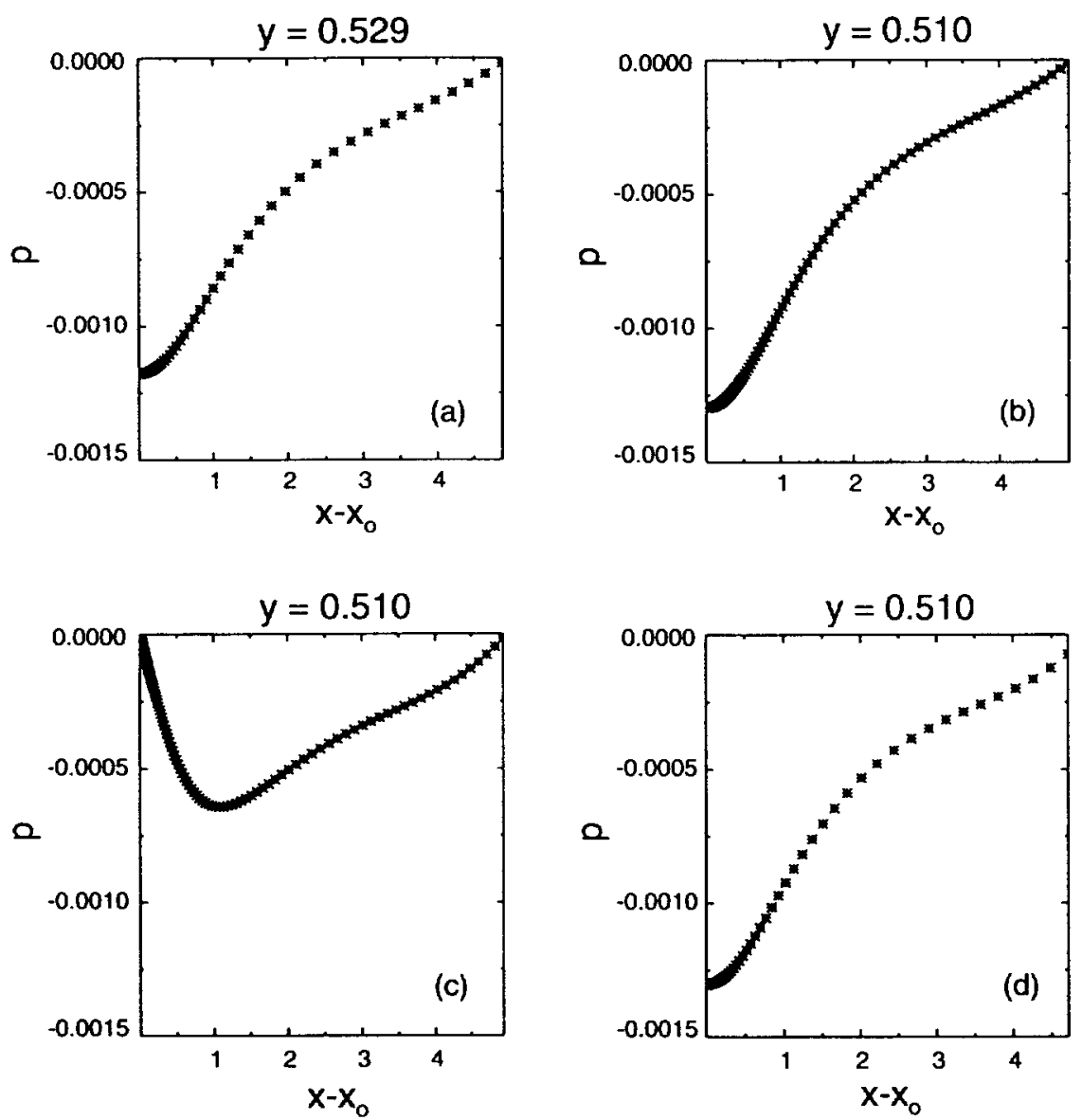

Figure 2.6. Pressure vs. $x$ at $y \approx 0.5$ for the (a) Run 1, (b) Run 2, (c) Run 3, and (d) Run 4. 


\subsection{Flow Over a 2-D Circular Cylinder}

The problem of two-dimensional flow about a circular cylinder has been studied quite extensively with experimental, computational, and theoretical techniques. This simple geometry produces a rather complex wake, which makes it a good test case for code benchmarking. For a Reynolds number, $\operatorname{Re}_{d}$, greater than $4\left(\operatorname{Re}_{d}=U d / v\right.$, where $U$ is the freestream velocity, $d$ the cylinder diameter, and $v$ the kinematic viscosity of the fluid), the flow about the cylinder separates off of the cylinder's aft face, resulting in two counter-rotating vortices in the immediate wake. When $R e_{d}$ is increased beyond $60-100$, these vortices alternately shed from the cylinder, giving rise to a wake consisting of counter-rotating vortices.

\subsection{Computational Setup}

The simulations of two-dimensional flow about a circular cylinder are conducted with the incompressible flow model in ALE3D. ${ }^{1}$ Two cylindrical grids (Figure 3.1) are employed for these simulations to determine the sensitivity of the results to the grid resolution. The chosen diameter, $d$, of the cylinder is 1 and the maximum radial dimension of both grids is $32 d$. To resolve the steep velocity gradients near the cylinder surface and in the cylinder wake, the computational grids are graded in both the radial and azimuthal directions. Table 3.1 lists the details of both grids.

The inlet boundary conditions are such that $(u, v)=(1,0)$ for $r=32 d$ and $90^{\circ} \leq \theta \leq 270^{\circ}$, where $u$ and $v$ are the horizontal and vertical velocity components, respectively. A zero natural boundary condition ${ }^{\prime}$ is imposed at the outlet $\left(r=32 d,-90^{\circ} \leq\right.$ $\left.\theta \leq-90^{\circ}\right)$. Earlier simulations demonstrated that significant flow reversal can occur at the outlet when the flow is well resolved there. Changing either the integration to full Gaussian quadrature or turning hourglass correction on had no effect in eliminating the flow reversal at the outlet. Only coarsening of the grid at the outlet appears to reduce this phenomena from occurring. The mechanisms for this unusual and erroneous flow reversal have not been investigated at the present time. In the subsequent simulations which have a relatively coarse grid at the outlet, this phenomenon is not observed in the flow field. However, this phenomenon may still have some small influence on the calculated results. The out-of-plane velocity component, $w$, is set to 0 for all nodes and the surface of the cylinder has a no-slip/no-penetration boundary condition $(u=v=0)$. At $t=0$, the entire flow is initialized with a velocity of $(u, v)=(1,0)$. The code then projects this initial condition to a divergence-free field. ${ }^{4}$ To reduce the computational time required for the flow to behave in a quasi-periodic manner, a random velocity perturbation is added to the flow about the cylinder at $t=0$. Both the CFL number and the diffusion stability number are set to 0.3 for both grids. In the subsequent simulations, the time steps for both coarse and medium grids are diffusion limited. The kinematic viscosity of the fluid is taken to be $v=0.001$, yielding a $R e_{d}=1,000$. The ALE3D input file for these simulations is shown in Appendix 2. The governing equations are solved with a conjugate gradient solver with a tolerance set to $10^{-8}$. The explicit time integration scheme is used for both grids. The simulations are run to a point at which the measured 
quantities, such as the lift and drag on the cylinder, are behaving in a repeatable manner as the vortices are shed from the cylinder. For the coarse grid, this means running the simulation up to $t \approx 900 \mathrm{~s}$ and, for the medium grid, until $t \approx 275 \mathrm{~s}$. Post-processing of these simulations is done with Interactive Data Language (IDL) of Research Systems, Inc.

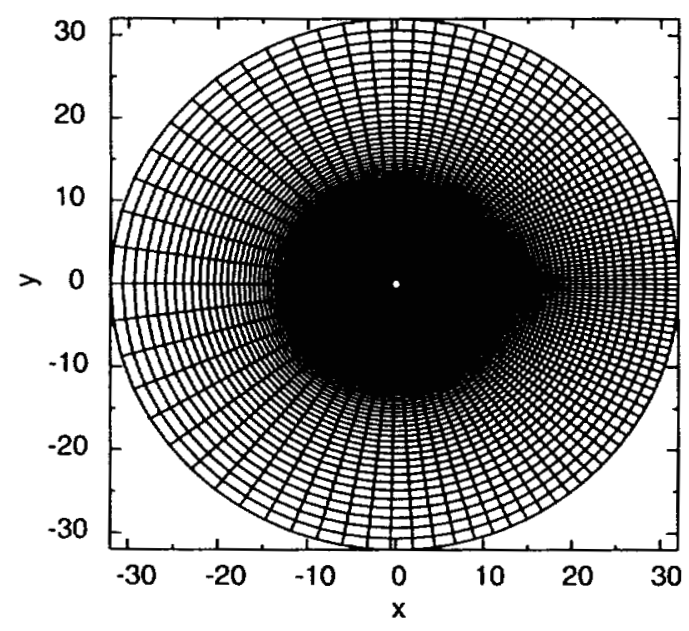

(a)

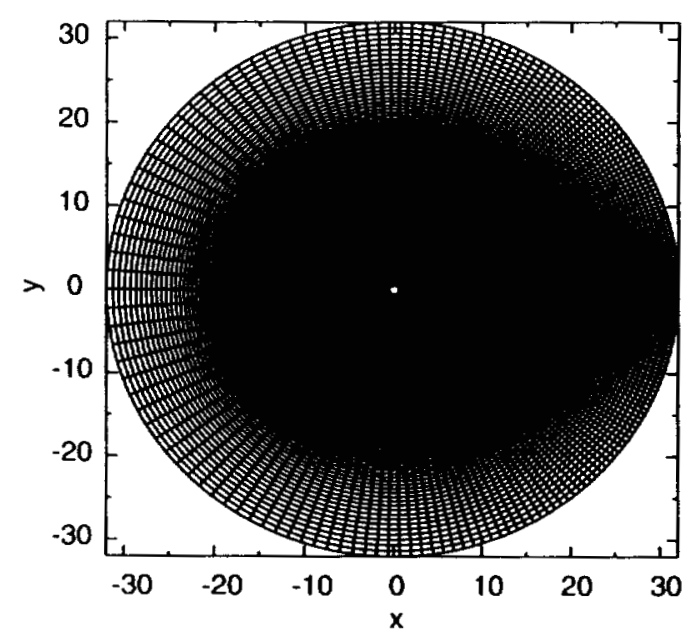

(c)

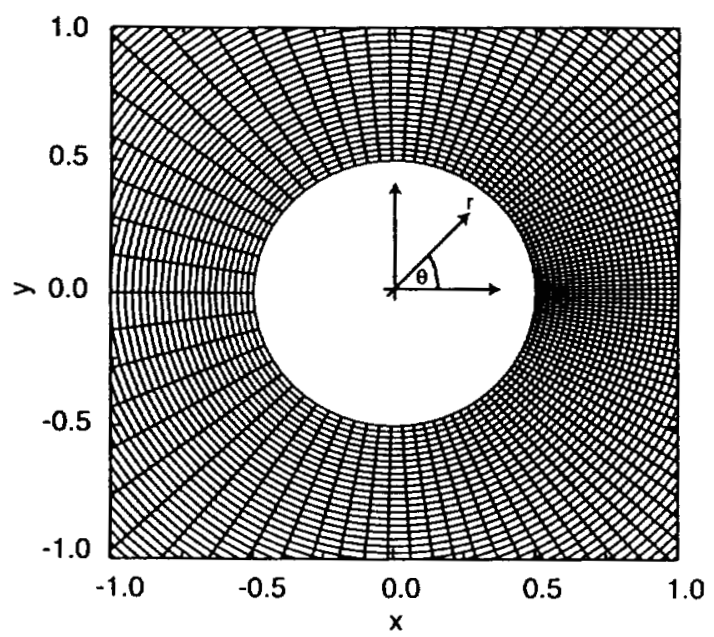

(b)

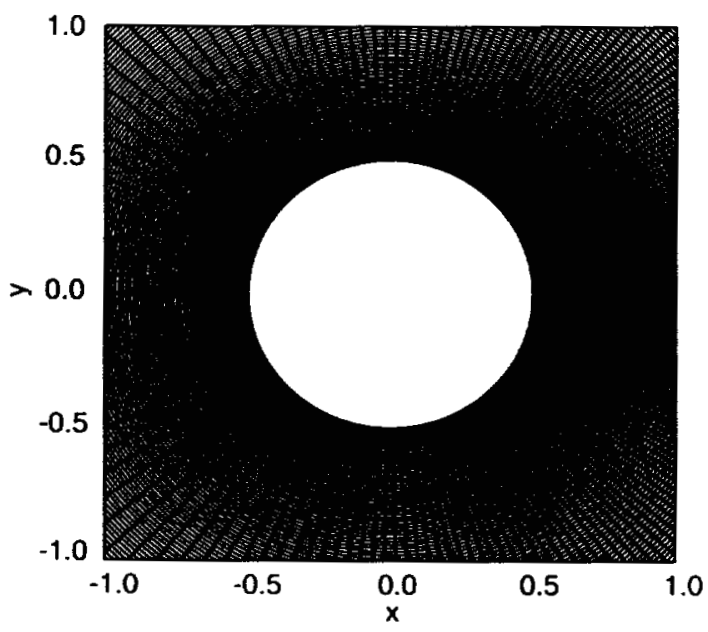

(d)

Figure 3.1. Coarse (a) and medium (c) grids used to simulate the flow about a circular cylinder. Close-up views of the (b) coarse and (d) medium grids. 


\begin{tabular}{|c|c|c|c|c|c|}
\hline Grid & $\begin{array}{c}\text { Total } \\
\text { Number of } \\
\text { Elements }\end{array}$ & $\begin{array}{c}\text { Number } \\
\text { of } \\
\text { Elements } \\
\text { in } r\end{array}$ & $\begin{array}{c}\text { Number } \\
\text { of } \\
\text { Elements } \\
\text { in } \theta\end{array}$ & $\Delta \mathrm{r}_{\min }$ & $\Delta \theta_{\min }\left(^{\circ}\right)$ \\
\hline Coarse & 20,000 & 200 & 100 & 0.02169838 & 1.1638158 \\
\hline Medium & 80,000 & 400 & 200 & 0.01082214 & 0.5764392 \\
\hline
\end{tabular}

Table 3.1. Details of the coarse and medium grids

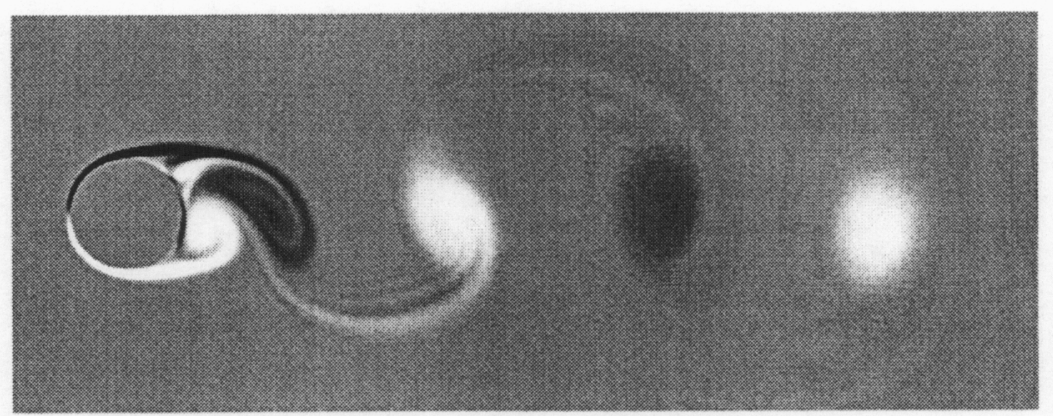

Figure 3.2. Instantaneous vorticity field about the cylinder for the medium grid. The white color levels indicate positive vorticity and the black color levels negative vorticity.

\subsection{Results and Discussion}

Figure 3.2 shows an instantaneous vorticity field about the cylinder. The pattern of vortices with alternating senses of rotation in the wake is clearly visible in this figure. This periodic shedding of vorticity results in a periodic variation in both the lift and drag on the cylinder, with the drag varying twice as quickly as the lift. Figure 3.3 shows the drag coefficients $\left(C_{d}=D /\left(\frac{1}{2} \rho U^{2} d\right)\right.$, where $D$ is the drag force on the cylinder) due to pressure and shear forces throughout both simulations. Note that the total drag, which is the sum of the pressure and shear forces, is due primarily to the pressure forces. The vertical dashed lines in Figure 3.3 indicate the sampling period over which average quantities are calculated. For both grids, this period corresponds to approximately 40 shedding cycles. Figure 3.4 shows the variation of the drag versus time over a small portion of the simulation.

Two important quantities are the average values of the drag coefficient, $C_{d}$, and the lift coefficient, $C_{l}=L /\left(\frac{1}{2} \rho U^{2} d\right)$, where $L$ is the lift force on the cylinder. The average values of $C_{d}$ and $C_{l}$ are plotted in Figure 3.5 as functions of the number of samples, $N$, made over the sampling period. It can be seen that for both grids, the amplitude of variation in $C_{d}$ and $C_{l}$ decreases with increasing $N$. Theoretically, $C_{d}$ and $C_{l}$ should become independent of $N$ as $N \rightarrow \infty$. However, it can be seen that small 
amplitude oscillations exist in the average values of $C_{d}$ and $C_{l}$ near the end of the sampling period. For the coarse grid, the final amplitude of oscillation in $C_{d}$ is approximately 0.0005 and that of $C_{l}$ approximately 0.006 . For the fine grid, the final amplitude of oscillation in $C_{d}$ is approximately 0.0005 and that of $C_{l}$ approximately 0.007 . These oscillations are likely due to the fact that the sampling period is not an integer multiple of the shedding period of vortices in the wake of the cylinder. Further reduction in the amplitude of the oscillation in the average values of $C_{d}$ and $C_{l}$ may be achieved by making the sampling period an integer multiple of the shedding period or by increasing the time of the sampling period.

The average values of $C_{d}$ and $C_{l}$ are listed in Table 3.2. Table 3.3 shows drag coefficients and Strouhal numbers found in the literature for two-dimensional flow about a circular cylinder at $R e_{d}=1,000$. Comparing $C_{d}$ in Tables 3.2 and 3.3 demonstrates that the average value of $C_{d}$ from the medium grid is in fair agreement with the values of $C_{d}$ from the other more recent works on this topic. The average value of $C_{l}$ should equal zero since, on average, the cylinder generates no net circulation. Although $C_{l} \neq 0$ in these simulations, the magnitude of $C_{l}$ is much less than 1 . As discussed in the previous paragraph, running these simulations for a longer period of time or making the sampling period an integer multiple of the shedding period would further reduce the magnitude of $C_{l}$. Taking the FFT of $C_{l}$ over the sampling period allows for the calculation of the shedding frequency, $f$, from which the Strouhal number, $S t=f d / U$, can be obtained.

Table 3.2 lists the $S t$ for both the coarse and medium grids. There is a $4.6 \%$ change in $S t$ in going from the coarse to the medium grid. The value of $S t$ from the medium grid compares favorably with those from other works (Table 3.3).


Figure 3.3. Drag coefficients for the (a) coarse and (b) medium grids. $C_{d p}$ (upper black line) is the drag coefficient due to pressure forces and $C_{d s}$ (lower gray line) is that due to shear forces. 


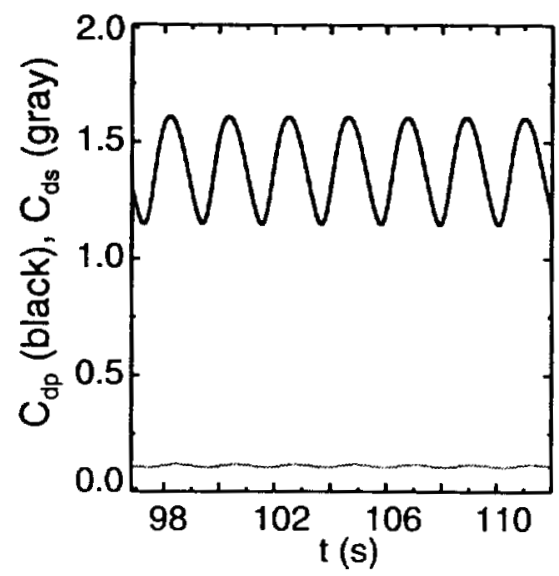

Figure 3.4. Drag coefficients for the medium grid over a small portion of the simulation. $C_{d p}$ (upper black line) is the drag coefficient due to pressure forces and $C_{d s}$ (lower gray line) is that due to shear forces.
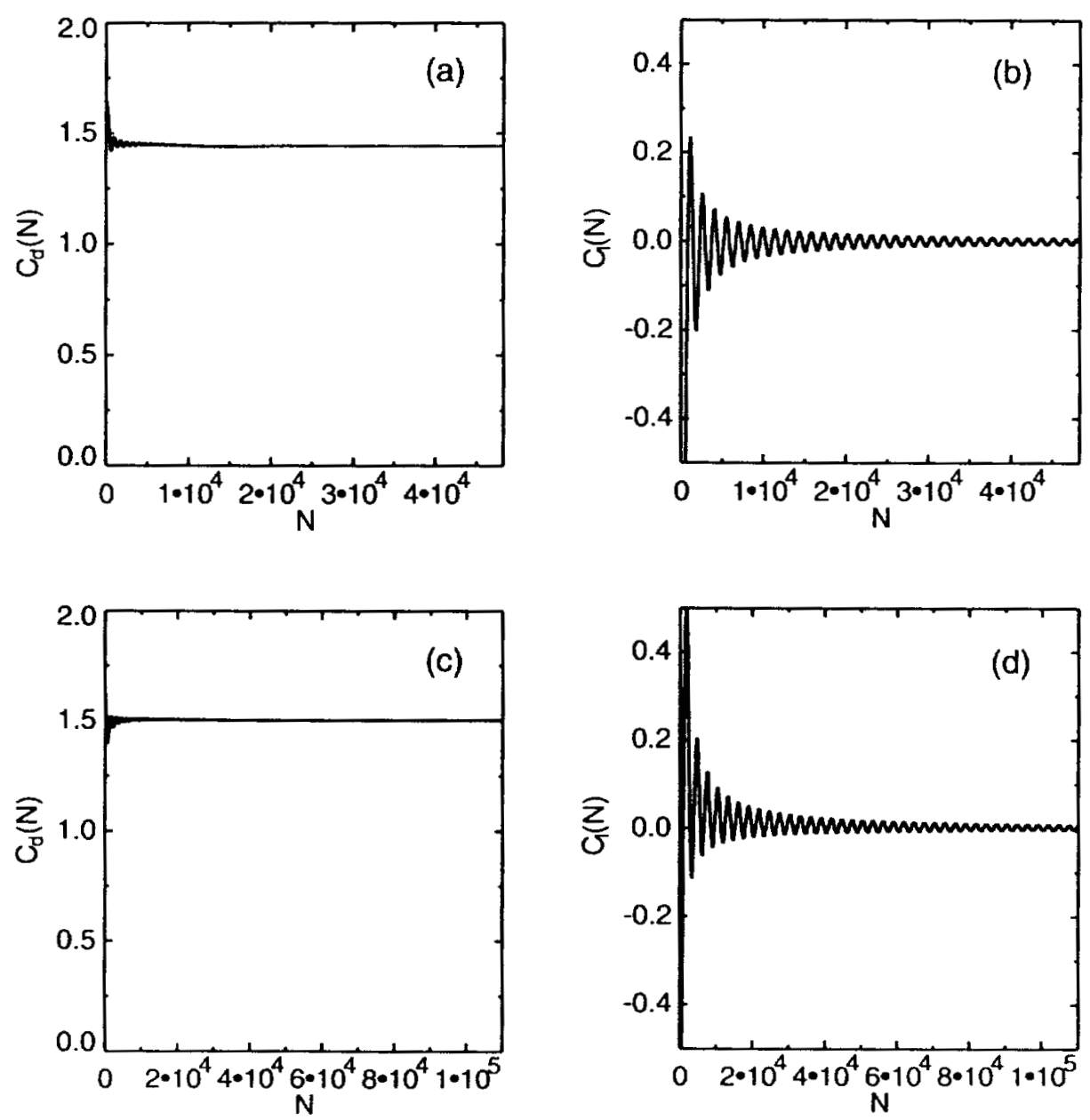

Figure 3.5. Variation of $C_{d}$ and $C_{l}$ with the number of samples, $N$, made during the sampling period for the (a-b) coarse grid and (c-d) medium grid. 
An examination of the average flow field in the wake of the cylinder reveals the streamline patterns shown in Figures 3.6-3.7 for the coarse and medium grids. Qualitatively, the average streamlines for both grids appear very similar: the flow forms two separation points, one reattachment point, and three recirculation zones on the upper and lower halves of the cylinder. To provide a more quantitative comparison between the average wake structures for the coarse and medium grids, the separation angle, $\theta_{\text {sep }}$, and recirculation length, $l_{\text {circ }}$, are measured. The separation angle is defined to be the angle at which the oncoming flow first detaches from the cylinder, causing the wall shear stress to vanish. The recirculation length is defined to be the distance between the surface of the cylinder and the maximum $x$ value at which the horizontal velocity, $u(x, y=0)$, is identically 0 . Figure 3.8 shows a pictorial representation of $\theta_{\text {sep }}$ and $l_{\text {circ }}$. Table 3.4 shows the $\theta_{\text {sep }}$ and $l_{\text {circ }}$ for the coarse and medium grids. For the coarse grid, there is a distinct asymmetry in the separation angle off of the top and bottom of the cylinder. This may be due to an asymmetric average pressure gradient along the surface of the cylinder or to the possibility that the sampling period is not an integer multiple of the shedding period. This asymmetry in the separation angle is reduced for the medium grid. There is a substantial change in the separation angles $(-7.6 \%$ for the top separation angle and $\sim 10.3 \%$ for the bottom separation angle) and the recirculation lengths $(\sim 9.3 \%)$ when comparing the results from the coarse and medium grids. These differences may be an indicator that these simulations are not yet grid converged.

\subsection{Conclusions}

These benchmarking cases demonstrate the performance of the ALE3D incompressible flow model for flow over a flat plate and a two-dimensional circular cylinder. For the flat plate simulation, it is estimated that the implicit time integration scheme results in a twenty-four-fold reduction of the simulation time over that of the explicit time integration scheme. The smallest error in the horizontal velocity occurs for the case in which the implicit time integration scheme is used and the vertical velocity is not specified at the inlet. The errors in the vertical velocity are comparable for all four test cases. For the two explicit time integration cases with the flat plate, a problematic trend is observed. Since the errors in the velocity and shear stress are not significantly reduced through grid refinement, it may not be possible to further reduce the errors. Another problematic trend with the flat plate problem is the sensitivity of the velocity and shear stress to the outlet zero natural boundary condition. For the benchmarking case of a circular cylinder in which an explicit time integration scheme is employed, the drag coefficient and Strouhal number demonstrate convergence to a solution that compares favorably with results from other studies in the literature. Lastly, as these results are only for two specific flow problems, care must be taken when trying to draw conclusions for how well the incompressible flow model in ALE3D simulates other types of flows geometries at different $R e$. 


\section{Acknowledgements}

The author would like to thank Rose McCallen for running the flat plate simulations with the implicit time-integration scheme and Kambiz Salari for his helpful discussions in the preparation of this document.

\begin{tabular}{|c|c|c|c|}
\hline Grid & $C_{d}$ & $C_{l}$ & $S t$ \\
\hline Coarse & 1.4422 & 0.0021 & 0.2279 \\
\hline Medium & 1.5022 & 0.0067 & 0.2394 \\
\hline
\end{tabular}

Table 3.2. Measurements of the average drag coefficient, $C_{d}$, average lift coefficient, $C_{l}$, and Strouhal number, $S t$, from the ALE3D simulations.

\begin{tabular}{|c|c|c|}
\hline$C_{d}$ & $S t$ & Reference \\
\hline 1.52 & 0.24 & Qian \& Vezza $^{6}$ \\
\hline 1.5191 & -- & He et al. $^{7}$ \\
\hline 1.51 & -- & ${\text { Blackburn } \text { et al. }^{8}}^{{ }^{6}}$ \\
\hline 1.23 & 0.206 & ${\text { Cheng } \text { et al. }^{9}}^{10}$ \\
\hline 1.5091 & -- & Henderson $^{10}$ \\
\hline 1.53 & 0.241 & Behr et al. $^{11}$ \\
\hline 1.198 & -- & ${\text { Braza } \text { et al }^{12}}^{13}$ \\
\hline 1.43 & -- & Phuoc Loc $^{13}$ \\
\hline 1.2 & 0.21 & Roshko $^{14}$ \\
\hline
\end{tabular}

Table 3.3. Drag coefficient, $C_{d}$, and Strouhal number, $S t$, measurements for $R e_{d}=1,000$ from other studies of flow about a circular cylinder. 


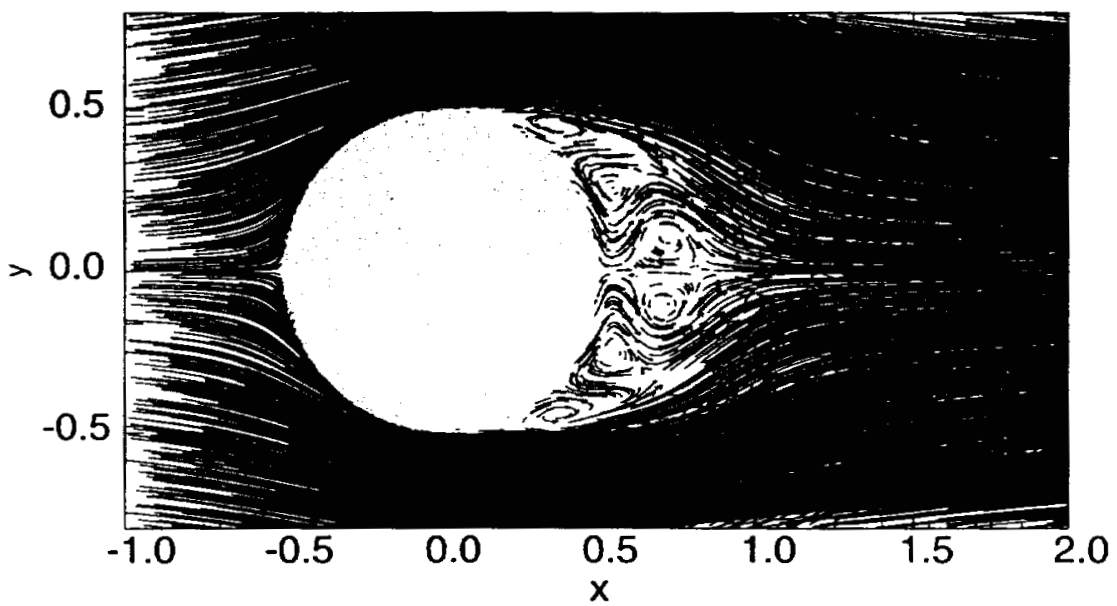

(a)

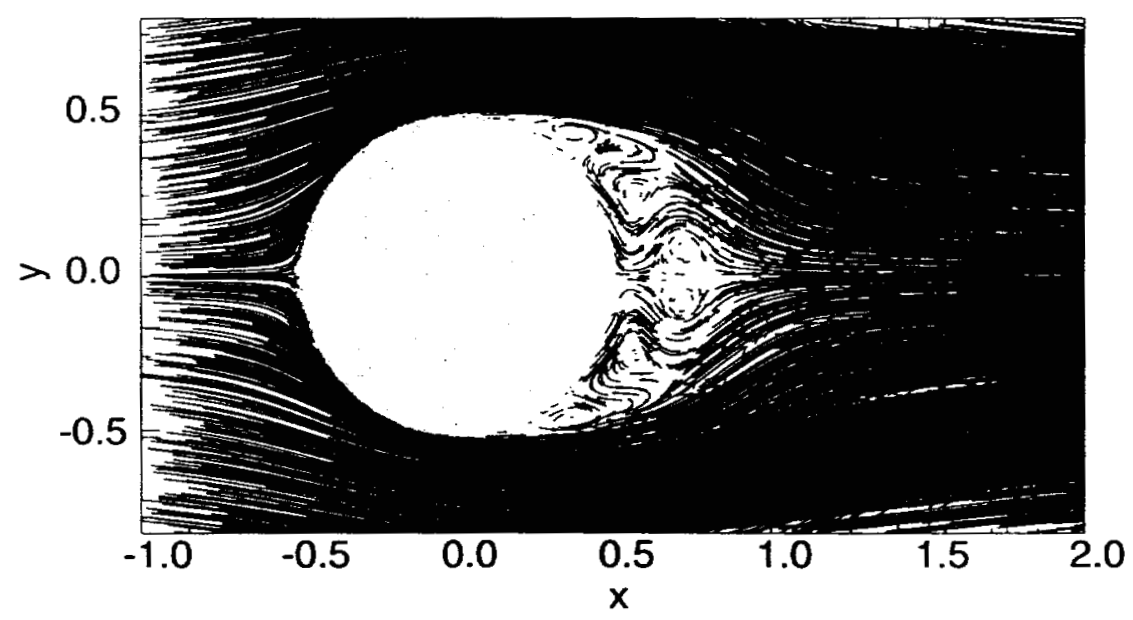

(b)

Figure 3.6. Streamlines of the average velocity field about the circular cylinder for the (a) coarse and (b) medium grids. 


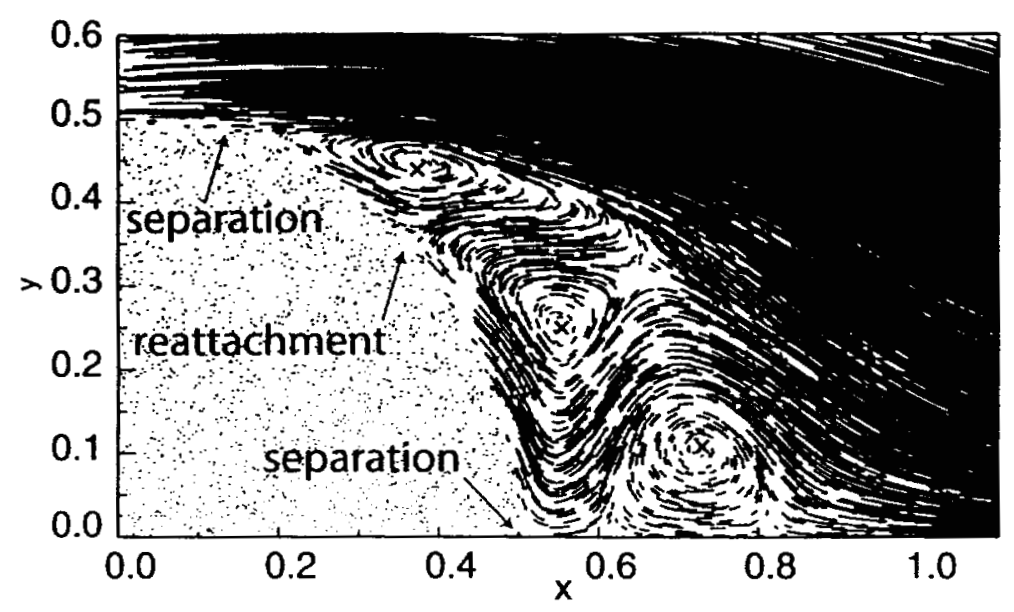

(a)

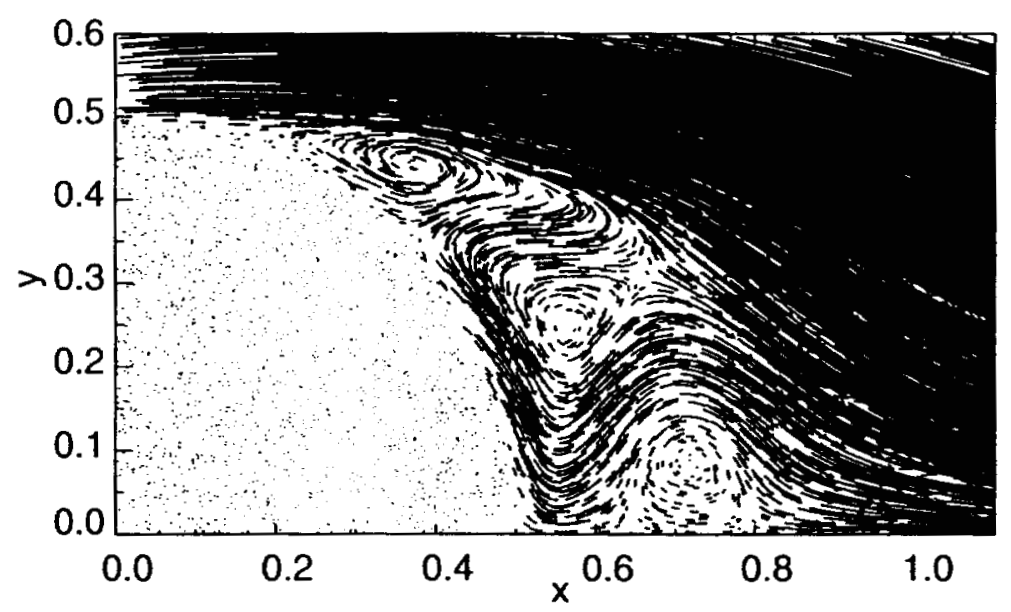

(b)

Figure 3.7. Close-up view of the streamlines for the (a) coarse and (b) medium grids. The $x$ 's in (a) indicate the approximate centers of the recirculation zones.

\begin{tabular}{|c|c|c|c|}
\hline Grid & $\theta_{\text {sep top }}\left({ }^{\circ}\right)$ & $\theta_{\text {sep bot }}\left({ }^{\circ}\right)$ & $I_{\text {circ }}$ \\
\hline Coarse & 66.084 & -64.728 & 0.290 \\
\hline Medium & 71.108 & -71.374 & 0.317 \\
\hline
\end{tabular}

Table 3.4. Separation angles and recirculation length measurements for the coarse and medium grids. 


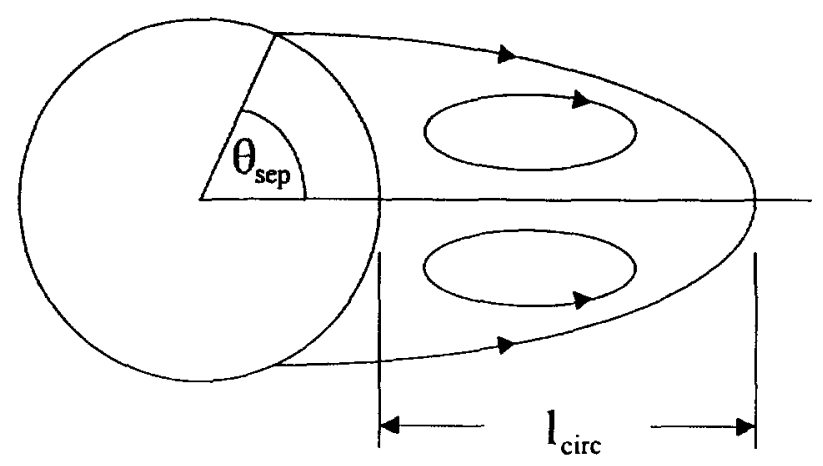

Figure 3.8. Separation angle and recirculation length definitions.

\section{References}

1. Dube, E., et al. 2001 User's Manual for ALE3D, Version 3.0.0, Lawrence Livermore National Laboratory.

2. Prandtl, L. 1904 Verhandlunger IIIrd. International Mathematiker Kongresser, Heidelberg, p. 484. Translation as NASA Memo 452.

3. Blasius, H. 1908 Z. Angew. Math. Phys., 56, p. 1 (Translation in NACA TM 1256).

4. Gresho, P.M. \& Sani, R.L., Incompressible Flow and the Finite Element Method, Wiley, 1998.

5. Dunn, T.A. \& McCallen, R.C., Parallel Computations of Natural Convection in a Tall Cavity Using an Explicit Finite Element Method, Int. J. Numer. Meth. Fluids, 2002, 3, 1-2.

6. Qian, L. \& Vezza, M. 2001 A Vorticity-Based Method for Incompressible Unsteady Viscous Flows, J. Comp. Phys 172, 515-542.

7. He, J.-W., Glowinski, R., Metcalfe, R., Norlander, A., \& Periaux, J. 2000 Active Control and Drag Optimization for Flow Past a Circular Cylinder, J. Comp. Phys. 163, 83-117.

8. Blackburn, H.M. \& Henderson, R.D. 1999 A Study of Two-Dimensional Flow Past an Oscillating Cylinder, J. Fluid Mech. 385, 255.

9. Cheng, M., Chew, T., \& Luo, S.C. 1997 A Hybrid Vortex Method for Flows over a Bluff Body, Int. J. Numer. Meth. Fluids 24, 253.

10. Henderson, R.D. 1997 Nonlinear Dynamics and Patterns in Turbulent Wake Transition, J. Fluid Mech. 352, 65.

11. Behr, M., Liou, R., Shih, R., \& Tezduyar, T.E. 1991 Vorticity-stream Function Formulation of Unsteady Incompressible Flow around a Cylinder: Sensitivity of the Computed Flow Field to the Location of the Out Flow Boundary, Int. J. Numer. Meth. Fluid 323. 
12. Braza, M., Chassaing, P., \& Minh, H.H. 1986 Numerical Study and Physical Analysis of the Pressure and Velocity Fields in the Near Wake of a Circular Cylinder, J. Fluid Mech. 165, 79.

13. Loc, T.P. 1980 Numerical Analysis of Unsteady Secondary Vortices Generated by and Impulsively Started Circular Cylinder, J. Fluid Mech. 100, 111-128.

14. Roshko, A. 1954 On the Development of Turbulent Wakes from Vortex Sheets, NACA Report 1191.

\section{Appendix 1 ALE3D Input Files for the Flat Plate}

Run 1: Coarse Grid, Explicit Time Step, $U_{\text {inlet }}$ and $V_{\text {inlet }}$ Specified

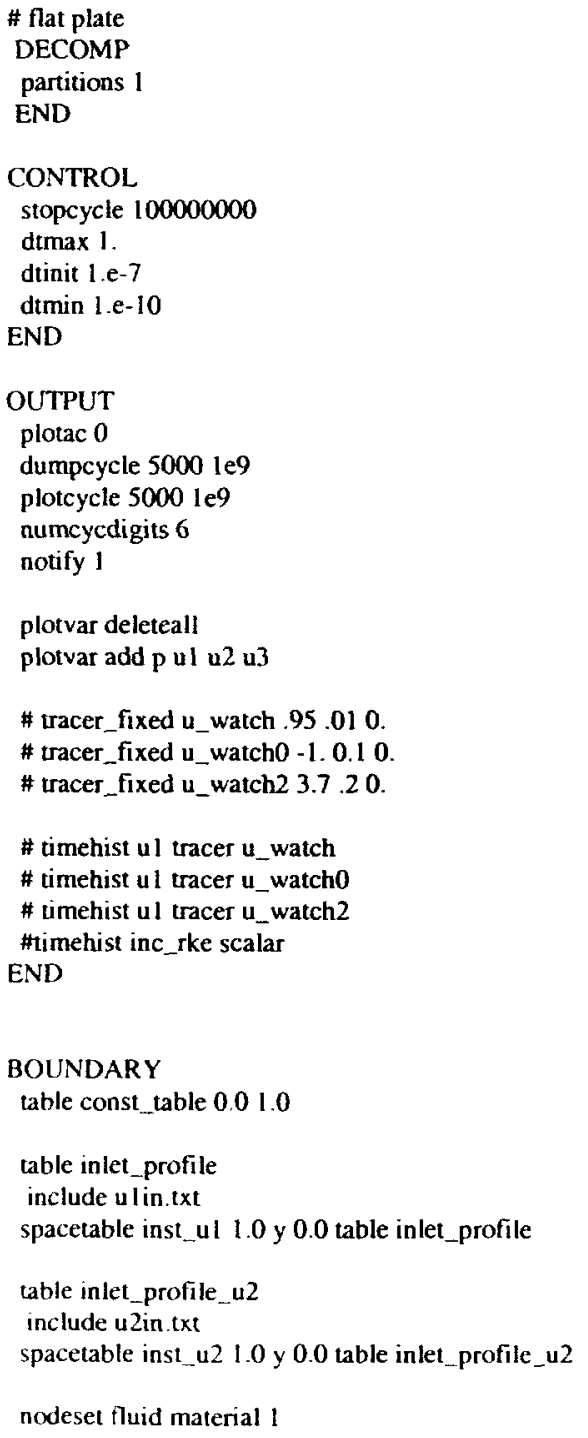


incvelocity fluid 1.00 .00 .0

incvel_loadcurve inletul spacetable inst_ul 1.01 .00 .00 .01 table const_table

incvel_loadcurve inletu2 spacetable inst_u2 1.00.01.00.01 table const_table

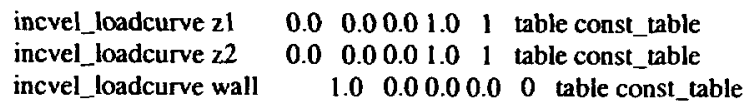

MATERIAL a 1

incinput tho 1. p0 $0.0 \mathrm{mu} 0.0005$ itrb 0 END

Run 2: Medium Grid, Explicit Time Step, $U_{\text {inlet }}$ and $V_{\text {inlet }}$ Specified

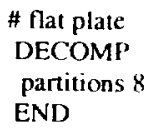


CONTROL

stopcycle 100000000

dtmax 1.

dtinit 1.e-7

dtmin 1.e-10

END

OUTPUT

plotac 0

dumpcycle 5000 le9

plotcycle 5000 le9

numcycdigits 6

notify 1

plotvar deleteal!

plotvar add p ul u2 u3

\# tracer_fixed u_watch .95 .010 .

\# tracer_fixed u_watch0 -1.0.10

\# tracer_fixed u_watch2 3.7.20.

\# timehist ul tracer u_watch

\# timehist ul tracer u_watch0

\# timehist ul tracer u_watch2

\#timehist inc_rke scalar

END

BOUNDARY

table const_table 0.01 .0

table inlet_profile

include $\mathrm{u}$ lin.txt

spacetable inst_ul 1.0 y 0.0 table inlet_profile

table inlet_profile_u2

include u2in.txt

spacetable inst_u2 1.0 y 0.0 table inlet_profile_u2

nodeset fluid material 1

incvelocity fluid 1.00 .00 .0

incvel_loadcurve inletul spacetable inst_ul $1.0 \quad 1.00 .00 .0 \quad 1$ table const_table

incvel_loadcurve inletu2 spacetable inst $u 21.00 .01 .00 .01$ table const_table

incvel_loadcurve zl $\quad 0.0 \quad 0.00 .01 .0 \quad 1$ table const_table incvel_loadcurve $z 2 \quad 0.0 \quad 0.00 .01 .0 \quad 1$ table const table incvel_loadcurve wall $\quad 1.0 \quad 0.00 .00 .0 \quad 0$ table const_table

END

HYDRO

nohydro 1

END

INCFLOW

inclogiterations

incstabflag 0

incstabcoef 0.01

inccfl 0.5

inccflv 0.5

inclumpmass 1 


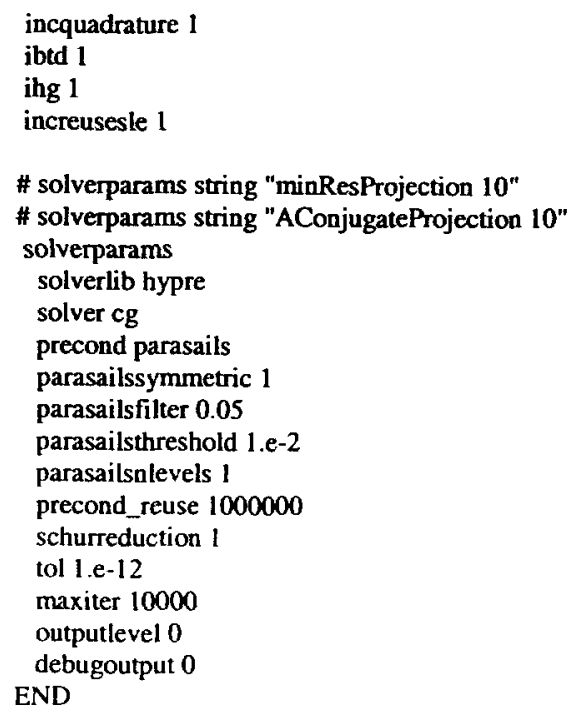

Run 3: Medium Grid, Implicit Time Step, $U_{\text {inlet }}$ Specified, $V_{\text {inlet }}$ Unspecified

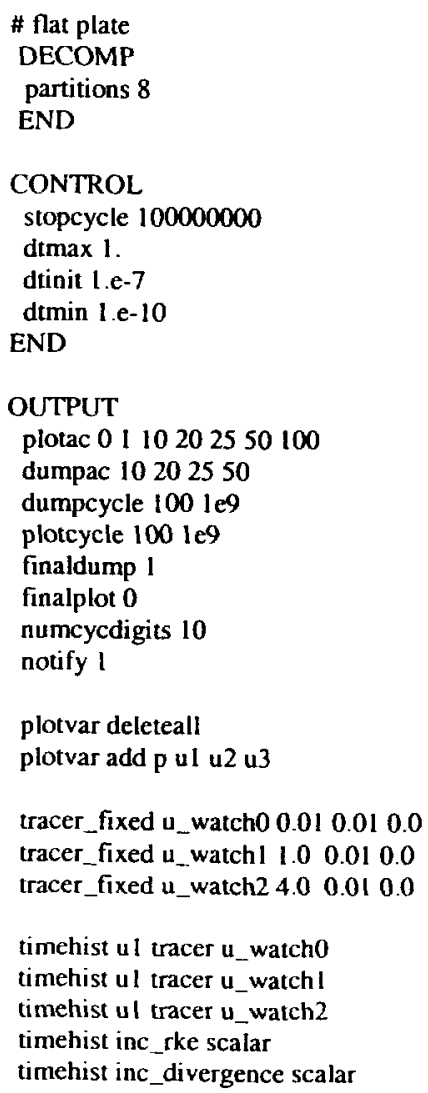




\title{
BOUNDARY
}

table const_table 0.01 .0

table inlet_profile

include ulin.txt

spacetable inst_ul 1.0 y 0.0 table inlet_profile

nodeset fluid material 1

incvelocity fluid 1.00 .00 .0

incvel_loadcurve inletul spacetable inst_ul $1.0 \quad 1.00 .00 .0 \quad$ I table const_table

\begin{abstract}
incvel_loadcurve zl $\quad 0.0 \quad 0.00 .01 .0 \quad$ I table const_table incvel_loadcurve z $20.0 \quad 0.00 .01 .0 \quad 1$ table const_table incvel_loadcurve wall $\quad \begin{array}{llllll}1.0 & 0.0 & 0.0 & 0.0 & 0 & \text { table const_table }\end{array}$
\end{abstract}

END

HYDRO

nohydro 1

END

NCFLOW

\# parameters needed for 2D implicit runs

incmethod implicit

inctime 1

\# for $2 \mathrm{~d}$ problems

inc2d 1

\# thetak and thetan (if both 1, then fully implicit)

\# note: if fully implicit, solve velocity with gmres (nonsym)

\# if semi implicit, OK to use cg for velocity (sym)

if thetak 1.0

ifthetan 1.0

\# The diffusion courant limit is set to a big number

incefl 3.

inccflv $1.0 \mathrm{e} 6$

inclumpmass 0

\# btd is turned off but may be needed for some cases

ibtd 0

ebtd 0.375

incresetppeguess 0

\# This makes sure you don't use the u-guess for $v$ etc

\# but instead use the previous values

incresetvelguess 1

inclogiterations 1

incstabflag 0

incstabcoef 0.01

\# We want quadrature to be 8 for implicit

incquadrature 8

incdivcalc 1

irke 1

incdivfree 1

\# Solver params needed for implicit solve

solverparams ppe string "minResProjection 10"

solverparams ppe

solverlib hypre

schurreduction I

solver $\mathrm{cg}$ 


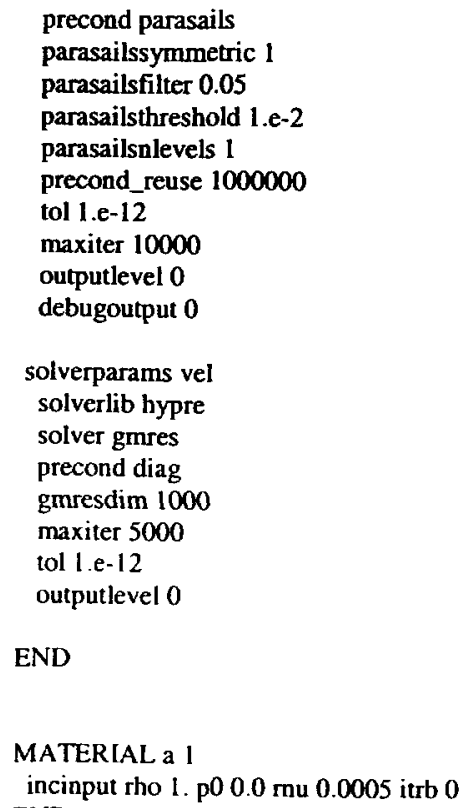

Run 4: Medium Grid, Implicit Time Step, $U_{\text {inlet }}$ and $V_{\text {inlet }}$ Specified

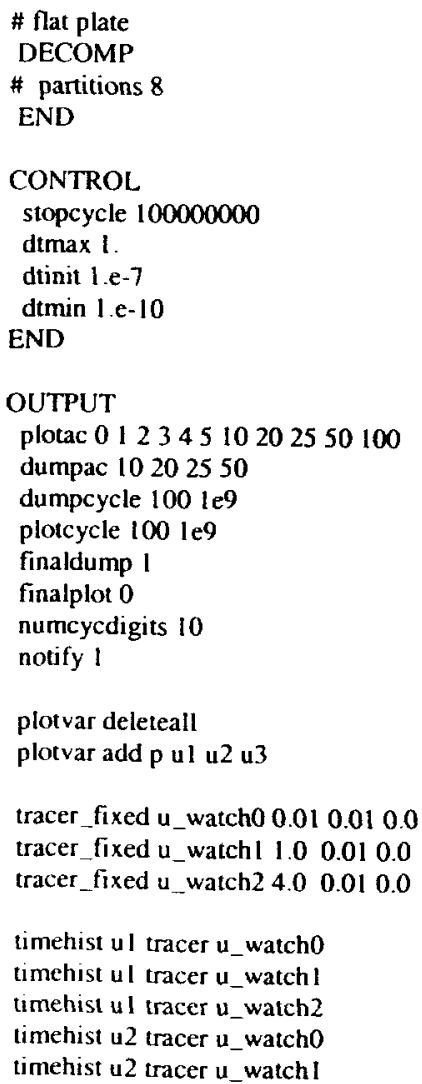




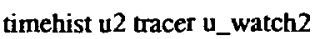

timehist inc_rke scalar

timehist inc_divergence scalar

END

\section{BOUNDARY}

table const_table 0.01 .0

table inlet_profile

include $u$ lin.txt

spacetable inst_ut 1.0 y 0.0 table inlet_profile

table inlet_profile_u2

include $u 2$ in.txt

spacetable inst_u2 1.0 y 0.0 table inlet_profile_u2

nodeset fluid material 1

incvelocity fluid spacetable inst_ul 1.00 .00 .0

incvel_loadcurve inletul spacetable inst_ul $1.0 \quad 1.00 .00 .01$ table const_table

incvel_loadcurve inletu2 spacetable inst_u2 1.00.01.00.0 I table const_table
increl loadcurve $\mathrm{zl}$
$0.0 \quad 0.00 .01 .0 \quad 1$ table const table
incvel_loadcurve $z 2$
$0.0 \quad 0.00 .01 .0 \quad 1$ table const_table
increl_loadcurve wall
$1.0 \quad 0.00 .00 .0 \quad 0$ table const_table

END

HYDRO

nohydro 1

END

INCFLOW

\# parameters needed for 2D implicit runs

incmethod implicit

inctime 1

\# for $2 \mathrm{~d}$ problems

inc2d 1

\# thetak and thetan (if both 1 , then fully implicit)

\# note: if fully implicit, solve velocity with gmres (nonsym)

\# if semi implicit, OK to use $\mathrm{cg}$ for velocity (sym)

ifthetak 1.0

ifthetan 1.0

\# The diffusion courant limit is set to a big number

inccfl 3.

inceflv $1 . e 6$

inclumpmass 0

\# btd is turned off but may be needed for some cases

ibtd 0

ebtd 0.375

incresetppeguess 0

\# This makes sure you don't use the u-guess for v etc

\# but instead use the previous values

incresetvelguess 1

inclogiterations 1

incstabflag 0

incstabcoef 0.01

\# We want quadrature to be 8 for implicit

incquadrature 8

incdivcalc 1

irke I 


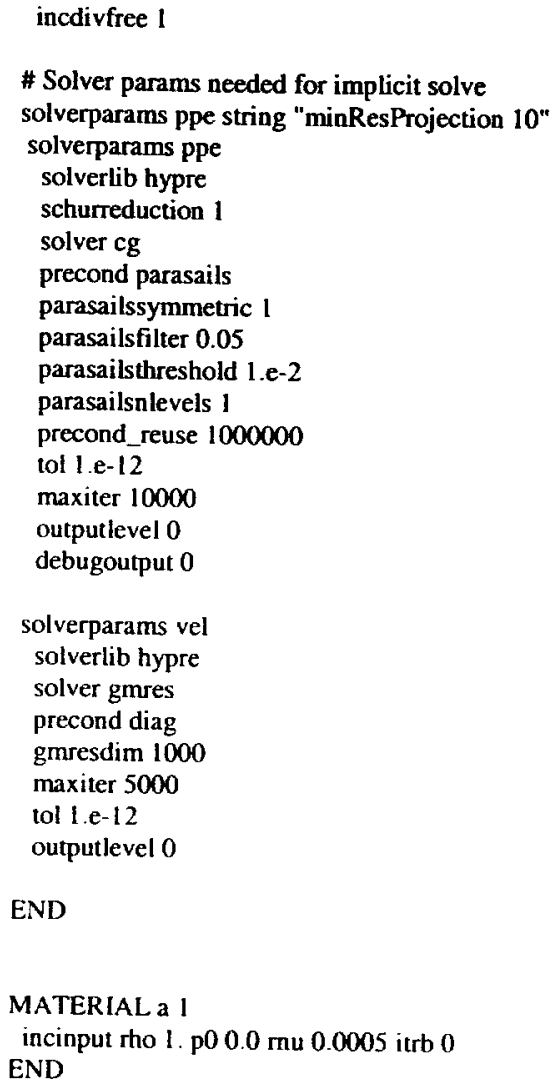

\section{Appendix 2 ALE3D Input File for the Circular Cylinder}

Coarse and Medium Grids

\# circle

DECOMP

partitions 16

END

CONTROL

stopcycle 1000000

\#stoptime 10.0

dtmax 1

dtinit 1.e-5

dtmin $1 . e-10$

END

OUTPUT

plotac 0

\#plottime 0.1 1.eg

\#finaldump I

\#finalplot 0

dumpcycle 5000 le9

plotcycle 2000 le9

numcycdigits 6

notify 10 
derivedvar drage nodeset prl scale 1. inc_fx

derivedvar liftc nodeset prl scale 1 . inc_fy

derivedvar dragp nodeset prl scale 1. inc_fxp

derivedvar liftp nodeset prl scale 1. inc_fyp

derivedvar drags nodeset prl scale 1 . inc_fxs

derivedvar lifts nodeset pr1 scale 1 . inc_fys

timehist_truncrestart 0

timehist dragc sum

timehist liftc sum

timehist dragp sum

timehist liftp sum

timehist drags sum

timehist lifts sum

timehist inc_rke scalar

timehist inc_divergence scalar

tracer_fixed v_time 04.00 .00 .0

timehist $\mathrm{u} 2$ tracer $v_{-}$time 0

tracer_fixed p_time 00.50 .00 .0

timehist $p$ tracer $p_{-}$time 0

plotvar deleteall

plotvar add $\mathrm{p}$ u $1 \mathrm{u} 2 \mathrm{u} 3$

plotvar add incta_u incta_v incta_p

plotvar add uvortz

END

BOUNDARY

table const_table 0.01 .0

nodeset lefthalf boundingbox $-100 .-100 .-100.0 .100 .100 .1 . e-2$

nodeset inlet intersection pr 2 lefthalf

nodeset blob filledcyl 0. 0. - 100. 0.0. 100.1. 1.e-1

incvel_loadcurve inlet $\quad 1.0 \quad 1.00 .00 .0 \quad 0 \quad$ table const_table

incvel_loadcurve prl $\quad 0.0 \quad 1.01 .01 .0 \quad 1$ table const_table

incvel_loadcurve pzl $\quad 0.0 \quad 0.00 .01 .0 \quad 1$ table const_table

incvel_loadcurve pz2 $\quad 0.0 \quad 0.00 .01 .0 \quad 1$ table const_table

incvelocity nali $1.00 .0 \quad 0.0$

incperturb blob 00.30 .30 .0

END

\section{INCFLOW}

incnodalforce 1

irke I

ivor 1

itavg u $v$

incdivcalc 1

inclogiterations 1

incstabflag 1

incstabcoef 0.01

incefl 0.3

inccflv 0.3

increusesle 1

solverparams string "minResProjection 10 "

solverparams

solverlib hypre

solver $\mathrm{cg}$

precond parasails

parasailssymmetric I

parasailsfilter 0.05

parasailsthreshold 1.e-2

parasailsnlevels 1

precond reuse 1000000

schurreduction I

tol 1 e- 8

maxiter 10000 
outputlevel 0

debugoutput 0

END

MATERIAL fluid 1

incinput tho $1.0 \mathrm{p} 00.0 \mathrm{mu} 0.001$

END 